\title{
Control of complexity in virtual organizations: the role of enterprise modelling
}

\author{
R.J. (Rob) Kusters and J.C. (Hans) Wortmann \\ Eindhoven University of Technology, Faculty of Technology Management, I\&T Department, \\ 5600 MB Eindhoven, the Netherlands.
}

\begin{abstract}
In general it can be stated that re-design and optimisation of a (virtual) enterprise is not facilitated but hindered by information systems. Too much of the 'way of working' of the existing situation is fixed in the existing systems to allow for easy changes. This problem is reinforced by the requirements for differentiation between similar but not identical business processes that further emphasize the need for increased flexibility, and the problems as caused by the current generation if enterprise information systems.

Adequate redesign of both the business processes of a (virtual) organization and the associated information systems requires enterprise modelling. In this paper we will, based on experiences gained in the area of product data modelling, develop a number of requirements for enterprise modelling methodologies, reference architectures, and languages. In this we will mainly focus on the areas of semantics, dynamics and complexity.
\end{abstract}

Keywords

Enterprise Modelling, Virtual Organisations, Ontologies, Life-Cycles.

\section{INTRODUCTION}

Virtual organizations are continuously engaged in changing their business processes. There are always opportunities to improve specific roles in the supply chain or in distribution channels. There are always reasons to change contracts with certain parties. These changes are often dependent on local circumstances. Therefore many changes in processes and roles are not implemented across the board, but in specific situations. This results in differentiation in processes, roles, contracts etc.

Due to this differentiation and continuous change, the complexity of virtual organizations is dramatically increasing. Mastering this complexity is not easy, and requires itself documentation. This documentation requires a language to describe the inter-organizational processes and responsibilities. Enterprise modelling provides such a language. This contention is supported by enterprise modelling literature, e.g. Vernadat [1996] and has lead to several developments in this area.

However, we are certainly not yet there. Enterprise Modelling Languages originated from practice, such as ARIS (Scheer [1994]) or DEM (Van Es and Post [1996]), do not have the concepts required to describe differentiated (context- 
sensitive) business processes. Reference Architectures such as CIMOSA (AMICE [1994]) do not describe the functioning of an enterprise in its business environment, and do not support change (except GERAM [Bernus and Geres, 1997] to some extend).

This paper aims to develop systematically some of the concepts required in enterprise modelling languages with the aim to align organization and ICT design. In order to proceed we looked at the design of (virtual) organizations and their supporting enterprise information systems as an engineering discipline. Any engineering discipline needs product data management (PDM) functionality for this purpose. Consequently, we approach the problem by studying the basic PDM concepts. In the following section, we start our analysis based on the literature. This is followed by our own analysis.

\section{LESSONS FROM PRODUCT DATA MANAGEMENT}

The problems described above are mainly related to the fact that ICT-support is required in a setting where several organizations are cooperating. This is a situation that exists commonly in complex product data development situations where engineers from different organizations and disciplines co-operate to develop a single product. The similarities between the two settings indicate that lessons from product development could be used in a virtual enterprise setting. Relevant literature in the area of product development can be found under the common denominator Product Data Management (PDM). In this section we will describe a frame of reference for PDM. This model was proposed by Den Hamer and Lepoeter (1996) and is aimed at the general problem of managing design data. The model distinguishes between five orthogonal dimensions (the 5-D framework), which are fundamental to design data management. The model is based on the experience of research groups within Philips in managing design data within ECAD, software development, end product / system development and mechanical $\mathrm{CAD}$ and has been in use for more then 10 years for analysing design data management issues. The five dimensions contained within the model are, when considered separately, each simple and readily recognizable to product developers. These five dimensions that will be described below are the version dimension, the views dimension, the hierarchy dimension, the status dimension, and the variants dimension.

\section{The version dimension}

A product design is not realized at once. Typically different designs will be looked at, and modifications and changes will be made. Also, after a product design has been finalized changes will still be made, e.g. to remove bugs or to add functionality. Each of these changes will result in a new version of the product design. This is no a linear process, often an older design will be used as the starting point for newer developments. Finalized designs can results in products being released to customers. For maintenance purposes it is required that the design data for these products remains available. For commercial reasons in the mean time (many) new versions will have been created. This implies that it is not sufficient to 
record a snapshot of the current situation but that data with regard to previous versions as well as the relationships between those versions need to be stored and made available.

\section{The views dimension}

Many products are simply too complex to represent in one single type of model. To deal with this typically multiple levels of abstraction are used. These are called 'views'. An example provided by Den Hamer and Lepoeter is: the classical electronic design process involves 1) the creation of electrical schematic, 2) the creation of a layout diagram, and 3) finally the generation of the data needed for production. This dimension is linked closely to the development process by which the views are derived from other views, either by full automatic transformation (e.g. compilation, format conversion) or by manual transformation into derived views.

This leads to two data management issues. The first refers to the fact that each view needs to be recorded separately, but is in it self relatively meaningless. Only the related set of views provides the information required. This requires recording and managing the links between related views.

The second data management issue deals with the information content of the different views. Although the views contain overlapping information this does not necessarily mean that one view can be derived from the other by a transformation. In the transformation often information is added (e.g. by adding details that previously did not exist). Errors can be made here and the semantic equivalence between the views can be lost. This requires careful monitoring and management of views, especially since the version dimension will intrude here. The existence of different versions implies the existence of different related sets of views which each have to meet with the semantic equivalence requirement.

\section{The hierarchy dimension}

Apart from using different design steps and thereby introducing different design views a commonly used technique is to decompose a design into smaller parts. This process can be repeated until the activities and their deliverables have become manageable. From a data management point of view, this means that the design data has a hierarchical structure where the relationships between the different elements of the hierarchy have to be managed. Note also that the hierarchy concept in it self might not pose too much problems but that it adds complexity to the data management issues as posed by the dimensions view and version.

\section{The status dimension}

In a design environment the information that is stable and tested is treated differently from information, which is untested and possibly incorrect. This 'status' dimension of design corresponds to the organizational procedures used to optimise design quality (e.g. verification, validation and release procedures). When design information passes such a step it results in a change of status. This does not mean that the information itself has changed; it means that the evaluation of the 
information has changed. This information is used to decide what can or can't be done with the information (e.g. unreleased information cannot be used to start production) and has to be managed and published carefully.

\section{The variants dimension}

This dimension is relevant when more then one variant of a particular product needs to be designed. The issue here is that these variants are to a large extend identical, but not identical. A data management issue here is the need to manage both the communality and the differences. Take as an example a freight truck, which consists of thousands of parts. The number of different trucks of a similar type that in principle can be ordered is huge. Explicit storage of the information describing all the possibilities is not feasible, even with modern database facilities. Data management needs to utilize communalities and identify differences in order to master this complexity. Note that variants and versions depict different concepts where versions are associated with evolution and variants with families of related products and as such a version can have many (even millions) variants, but a set of variants will belong to a single version.

\section{Discussion}

When looking at these dimensions a number of notes can be made. According to the authors these five dimensions are relevant to all design disciplines. They tested the ideas in Philips on mechanical design, circuit board design, IC design, software design, system design, and even multi media content design. Although the relative importance of items differs between design environments, each item appears to be meaningful in each of these areas. This means that a transfer to an environment in which e-commerce processes are designed and supported might be feasible.

Secondly we can notice that a number of the dimensions dealt directly with associated design processes. We saw a direct link between the dimension of view and the steps of the design process itself as well as a link between the dimension of status and the quality process of design approval and release. The dimension of version can be related to the process issue of design iteration, that of hierarchy to the process issue of sub projects and finally the dimension of variants can be linked to the process issue of development of product families.

Given that e-commerce processes differ from 'pure' design processes it is to be expected that revision of these dimensions is required before such a transfer into the setting of virtual organizations can be attempted. The first processes that spring to mind are those of sale and transport, both essential in a virtual enterprise ecommerce setting. We will include these two processes in the discussion below, but in general the message here is that identification of relevant cross- organizational processes and the effect of these processes on the enterprise model of the virtual enterprise has to be part of an enterprise modelling methodology.

A third and final note is that the 5-D framework focused explicitly on products and product data. In the setting of enterprise modelling aimed and designing and managing a virtual enterprise this restriction must be abandoned. Enterprise modelling aims at identification and data management of enterprise objects. An 
enterprise object is an abstract concept that can depict any relevant type of object that is of interest for a virtual enterprise. Products, process, functions, departments and resources are typical examples of enterprise objects that will play a part in enterprise modelling. Note that, given that this discussion aims at enterprise modelling as a means for designing and managing virtual enterprises, enterprise object models are also relevant enterprise objects that can be modelled and dealt with in similar ways as the more obvious enterprise objects such as products, processes and resources. We can e.g. identify versions and variants in enterprise models just as easily and just as usefully as we can identify versions and variants of products. In the 5-D framework the identified dimensions are applied only to products but the remainder of the paper we will apply these notions to the more general concept of enterprise object.

\section{Analysis}

In trying to apply the 5-D framework of Den Hamer and Lepoeter to the environment of virtual enterprise and e-commerce we will first take a closer look at the issues that are addressed by these five dimensions. When doing so we can identify three of these issues.

The first and most obvious of these is the issue of dynamics. Data management often assumes a somewhat 'static' situation. The data models in use can then be developed by as it were taking a 'snapshot' of this situation. If, e.g. because of an organizational change, changes are required these will result in a new design and a new 'snapshot' which is then implemented. History (the previous 'snapshot') can now be discarded because it is no longer relevant in the new situation.

Den Hamer and Lepoeter in their paper concluded that a dynamic environment as the product design environment required explicit handling of changes. The dimensions version, status and view are introduced to enable handling of dynamics efficiently.

We already concluded in previous paragraphs that in the setting of virtual enterprises and electronic commerce similar (but not identical) problems with handling dynamics exist. Based on this we will identify relevant dimensions for dealing with dynamics.

The second issue to be dealt with is that of complexity. Den Hamer and Lepoeter provided two dimensions that deal directly with complexity. These are hierarchy and variants. Hierarchy is introduced to master the complexity inherent to large product development effort by means of decomposing the product into components. As an added benefit of this approach the option of component reuse is presented. As a second means of mastering complexity the dimension of variants is introduced aimed at dealing with (a large degree of) product variation as is becoming more and more common practice in the setting of product family or platform development.

We can identify a similar (but again not identical) need to master complexity in the setting of virtual organizations. Identification of specific products can be as complex as in the setting of product design and specifically in the context of mass customisation process complexity is added as well. 
The third and final issue is that of semantics. Using the five dimensions explicitly results in a number of related models depicting versions, views, etc.. To be useful these models have to show semantic equivalence. Information presented in a model that also occurs in another model has to have an identical meaning to all the people who develop and / or use the models. This issue is not dealt with explicitly in the Hamer Lepoeter paper but is referred to in a footnote. When discussing the dimension 'views' the authors note that the view concept is closely related to (intended) semantic equivalence. In the footnote they explain that in case of information overlap the intention is to achieve semantic equivalence but that this cannot be guaranteed because of possible incorrect transformations between the views. In our view this is a structural problem that is not restricted to the views dimension. It has wider implications that deserve special treatment.

We now have identified three main requirements to which an enterprise modelling language and associate methodology has to possess:

a the ability to master differences in semantics

a the ability to master dynamics

- the ability to master complexity

We will now discuss each of these main requirements and in this fashion adapt the 5-D model to a setting of a virtual enterprise while in doing so develop a number of more detailed requirements to which enterprise modelling languages and methodologies should adhere if they are to provide the support that is needed for virtual organization.

\section{Mastering differences in semantics}

An enterprise model is the integrated set of models that together depict relevant parts and / or aspects of an enterprise. It is a set of models since no model by itself can capture the required width and depth of information that is required to run an enterprise. The models need to be integrated to insure consistency. The achievement of such consistency is not trivial, as we will illustrate.

A model is a description of (a part of) reality. However, a model will usually not depict an objective picture. The model will be developed on the basis of language. This language will be provided by a person (or group of persons) who will often use a specific jargon and who will have a specific purpose in mind when designing the model. When two different information systems are developed on the basis of two different models (or more likely: two different sets of models) and these two systems are supposed to exchange messages this will only be effective when both systems attach the same meaning to the contents of the message. This in its turn implies that the models that form the basis for these information systems agree as to the semantics of the messages involved. If different models are developed based on different languages (or jargon) is agreement need not occur automatically.

In this discussion two important items arise, that of '(group of ) persons' and that of 'purpose'. Any group of persons that cooperate in a specific area to a specific purpose tend to develop a language specific to that area and that group. Based on this language an ontology can be developed. The language (or jargon) usually exists implicit in the minds and behaviour of the persons involved. By identifying 
explicitly what is specific to this jargon an ontology can be developed. An ontology is in the terms of Gruber [1993] 'a specification of a conceptualisation', or, more pragmatic, a common set of definitions shared by a group of persons.

If enterprise models consist of models that are developed by a diverse set of groups of people model integration requires an explicit and formal recognition of the jargons used. Since jargons are by definition implicit this means that use of ontology is required here. Formal communication between groups of people that do no share the same jargon (and of models developed by them) requires taking account of the existence of ontologies for each group involved and the explicit development of ontological commitments. An ontological commitment can be defined as an agreement to use a vocabulary (i.e., ask queries and make assertions) in a way that is consistent (but not complete) with respect to the theory specified by an ontology (Gruber [1993]).

In any virtual enterprise where two or more real organizations cooperate it is logical to assume that each real organization will have a particular jargon, which have to be taking into account when developing the enterprise model for the virtual enterprise. However, it is likely that more relevant groups need to be identified. Professionals (e.g. electric engineers, lawyers) tend to have a discipline specific usage of language that is developed and maintained at educational institutes. People working together in an organizational department or communicating regularly in the context of a jointly executed organizational function of business process also tend to develop local language idiosyncrasies.

Note that this requirement does not imply that full ontologies for all relevant participating groups have to be developed. We are only interested in that part of the local language that is needed for communication with other groups in the context of enterprise modelling. If a local group uses a jargon consisting of hundreds of specific terms and only four of these are required for communication purposes in the setting of an enterprise model this implies that the ontological commitment required can be reduced to these four terms.

Based on this discussion an enterprise modelling methodology requirement can be identified. When developing models explicit identification of the specific groups involved in the modelling effort is needed, followed by the development of and agreement to the required ontological commitments.

\section{Mastering dynamics}

The second issue to be discussed further is that of mastering dynamics. Note that the 5-D framework uses the dimensions of version, view and status to handle this. When looking these can note two different foci. 'Status' looks at the different conditions a single enterprise object can exhibit. The other two look at relationships between different enterprise objects. We will take this division as a starting point in our discussion.

A single enterprise object can be seen to have different statuses. In identifying relevant statuses Den Hamer and Lepoeter looked at the different stadiums of a quality control process. If we abstract from this specific process we can state that any enterprise object can exhibit a life cycle in which different states and state transitions can be identified. 
From a quality point of views these states can e.g. be 'work in progress', ready for testing', 'tested', 'approved', 'released for production', and 'retired'. This quality point of view is an obvious choice to take during product development. This point of view stays valid in virtual enterprise development and management but can now also be applied to other enterprise objects such as the business processes of the virtual enterprise and its (shared) resources where similar quality concerns are relevant.

Apart from the quality process other process can be selected to at least to other ways of defining life cycles need to be looked at as well. As was noted above, two obvious candidates are 'sales' and 'transport'. If we take into account these processes we can identify two additional means of defining life cycle, namely that of ownership and location. In a virtual enterprise consisting of several legal entities it is essential to keep track of ownership of enterprise objects. This can be envisaged by a life cycle containing state transitions such as: legal transfer of ownership; transfer of financial obligation; transfer of money; transfer of physical object, etc.. Similarly keeping track of the location of the enterprise object is relevant. Here we can envisage states such as: in transit, in storage facility $\mathrm{X}$, etc. This life cycle approach is obviously applicable to products as was shown in the 5-D framework. It is easy to see that this approach is equally applicable to other type of objects such as resources, business processes, etc. So, instead modelling of a single life cycle model it is possible to identify several more or less independent life cycles. Depending on the types of processes that are included in a specific virtual enterprise other life cycle points of view can be included but three points of view discussed here (quality, sales, transport) are likely to exist in all virtual enterprises and should as such be included in enterprise modelling languages.

Instead of looking at a single enterprise object we can also study the dynamics involved when looking at a series of enterprise objects and enterprise object models. Here we are interested in the (dynamic) relationships that can exist between these objects. In the 5-D model the dimensions of version and that of view are introduced to deal with this aspect. These two dimensions can be looked at as two specific examples of a more generic dimension that we will call 'progeny'.

The notion of progeny describes the relationship between an enterprise object and all enterprise objects the preceded it in some way. The 5-D notion version in refers to the specific case where a product (or more general: an enterprise object) is the successor to a similar enterprise object. The 5-D notion of view refers to the specific case where a model is developed as a further refinement of a more general model. Important other types of progeny that can be envisaged are e.g. that of the relationship between a design and its resulting product and that of the relationship between various versions of models. In general a proper use of the progeny concept allows the description of causal relationships as well as relationships over time. This in its turn allows tracking of changes and assessment of the effect of proposed changes.

Mastering dynamics in this situation is closely linked to the requirement of flexibility. Flexibility refers to the ease with which changes can be made. This is a requirement for successful implementation of e-commerce processes in a virtual enterprise setting. If a virtual enterprise is to be organized or reorganized efficiently en effectively a working knowledge of all progeny relationships is required. This 
will enable identification of consequences of proposed changes and facilitate the design and implementation process thus furthering the required flexibility.

From the discussion above we can draw the conclusion that enterprise modelling languages and methodologies should incorporate the capability to handle (identify, model, manage, and maintain) life cycle models from different points of view (but certainly including quality, ownership, and location) as well as the ability to handle the various type of progeny described above.

\section{Mastering complexity}

The final issue to be discussed is that of mastering complexity. Den Hamer and Lepoeter already noted in their 5-D framework that product design is a very complex process and provided the dimensions of hierarchy and variant to help deal with this complexity. Given the instead of dealing with 'just' product data we are now dealing with wider enterprise data the degree of complexity will expand correspondingly ad so will the need for mastering this complexity.

In general the notions of hierarchy and variant can be seen as a specific case of abstraction. In traditional abstraction literature (Brodie e.a., [1984]) three types of abstraction are presented:

- Generalisation / specialisation. This is depiction of the IS-A relationship between enterprise objects. The relationship is formalized by the explicit identification of common parameters between different types of enterprise objects.

* Aggregation/decomposition. This is a depiction of the PART_OF relationship, which is equivalent to the hierarchy dimension described in the 5-D framework. The relationship is formalized by the explicit decomposition relationship between different types of enterprise objects.

a Grouping/ungrouping. This is a depiction of the SET_OF relationship between enterprise objects. The relationship is formalized by the explicit identification of the membership function between different types of enterprise objects.

- An additional mechanism is of more recent date (Hegge, [1995]).

* Parameterisation: This is a form of abstraction specifically aimed at managing the 5-D dimension of variants. The relationship is formalized by the explicit identification of common parameter values between different types of enterprise objects. This enables the implicit definition of (large) number of enterprise object variants.

Together these abstraction principles provide a powerful set of mechanisms that enable mastering of enterprise object complexity. These traditional abstraction mechanisms have proven their value in mastering complexity time and time again. Sufficient experience has been gathered using the parameterisation mechanism to prove its value as well and logic shows that this last mechanism is a useful addition since it allows us to master the complexity of instances of enterprise objects while abstraction allows us to master the complexity of enterprise object types. Together they provide a flexible use of parameters and parameter values to describe and structure complex enterprise objects. Concluding we would state that no enterprise modelling language should lack these modelling capabilities. 


\section{CONCLUSIONS}

Based on experiences in the area of product data modelling we identified a number of requirements that an enterprise modelling methodology and language should adhere to. These requirements focus on mastering semantics, dynamics and complexity.

\section{REFERENCES}

[1] AMICE (eds.), CIMOSA: Open Systems Architecture for CIM, Springer, Berlin, 1994

[2] Michael L. Brodie, John Mylopoulos and Joachim W. Schmidt (eds.), On conceptual modelling: perspectives from artificial intelligence, databases, and programming languages, Springer Verlag, Berlin, 1984.

[3] Bernus P. and Nemes L., Requirements of the Generic Enterprise Reference Architecture and Methodology, Annual Reviews in Control, Volume 21, 1997, Pages $125-136$.

[4] Gruber, T. R., A translation approach to portable ontologies, Knowledge Acquisition, 5(2):199 - 220, 1993.

[5] Hamer, P. van den and Lepoeter, K., Managing design data: the five dimensions of CAD frameworks, configuration management and product data management, Proceedings of the IEEE, vol. 84, nr. 1, Jan. 1996, pp. 42 - 56.

[6] Hegge, Herman M.H., Intelligent product family descriptions for business applications: production control software based upon generic bills-of-material in an assemble-toorder/make-to-stock environment, Ph.D.-Thesis, Eindhoven University of Technology, 1995.

[7] Scheer, A.W., Business Process Engineering, Springer, Berlin, 1994.

[8] Van Es, R.M. and Post, H.A., Dynamic Enterprise Modelling, Kluwer Bedrijfsinformatie, Deventer NL, 1996.

[9] Vernadat, F., Enterprise Modelling and Integration: Principles and Applications, Chapman \& Hall, 1996. 